
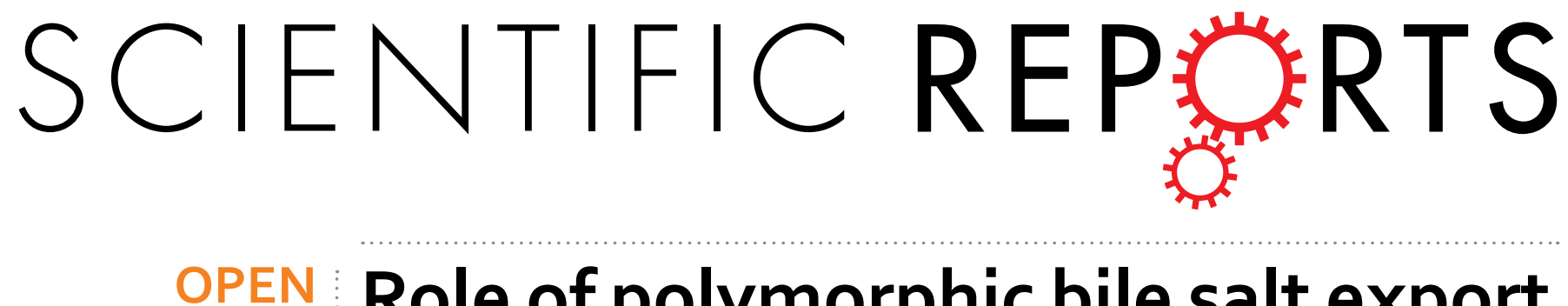

Received: 06 January 2016

Accepted: 23 May 2016

Published: 13 June 2016

\title{
Role of polymorphic bile salt export pump (BSEP, $A B C B 11$ ) transporters in anti-tuberculosis drug-induced liver injury in a Chinese cohort
}

Ru Chen ${ }^{1}$, Jing Wang ${ }^{1}$, Shaowen Tang ${ }^{2}$, Yuan Zhang ${ }^{3}$, Xiaozhen $\mathrm{Lv}^{4}$, Shanshan Wu ${ }^{1}$, Zhirong Yang ${ }^{1}$, Yinyin Xia ${ }^{5}$, Dafang Chen ${ }^{1}$ \& Siyan Zhan ${ }^{1}$

Evidence indicates that the polymorphisms in bile salt export pump (BSEP, encoded by $A B C B 11$ ) may play an important role in the development of anti-tuberculosis drug-induced liver injury (ATDILI) and we aim to investigate the association between genetic variants of $A B C B 11$ and the risk of ATDILI in a Chinese cohort. A total of 89 tuberculosis patients with ATDILI and 356 matched ATDILI -free patients constituted cases and controls. Genetic polymorphisms of $A B C B 11$ were determined by TaqMan singlenucleotide polymorphism (SNP) genotyping assay. Odds ratio (OR) with $95 \%$ confidence intervals (Cls) was estimated by conditional logistic regression model. There were no significant differences in genotype frequencies of $A B C B 11$ between cases and controls. In the subgroup analysis, polymorphisms of rs 2287616 were found to be associated with cholestatic/mixed pattern of liver injury under dominant and addictive model $(\mathrm{OR}=3.84,95 \% \mathrm{Cl}: 1.16-12.75, P=0.028$ and $\mathrm{OR}=2.51,95 \% \mathrm{Cl}: 1.12-5.62$, $P=0.025$, respectively), however the significance disappeared after Bonferroni correction. This study suggested that genetic variants of $A B C B 11$ gene might contribute to anti-tuberculosis drug-induced cholestatic liver injury in Chinese patients. Studies in larger, varied populations are required to confirm these findings.

The bile salt export pump (BSEP) which mainly located at the canalicular membrane of hepatocyte is an ATP-binding cassette (ABC) transporter encoded by the $A B C B 11$ gene. BSEP mediates the canalicular excretion of numerous bile salt into bile, which is regarded as the rate-controlling step of the vectorial transport of bile acids through hepatocytes ${ }^{1}$.

BSEP plays a key role in many liver diseases due to its crucial location and the important effects of bile acids ${ }^{2}$. Mutations of BSEP are known to cause cholestatic liver diseases of varying severity including progressive familial intrahepatic cholestasis ${ }^{3-5}$, benign recurrent intrahepatic cholestasis ${ }^{6,7}$ and intrahepatic cholestasis of pregnancy ${ }^{8,9}$, as well as drug-induced liver injury (DILI) ${ }^{10,11}$.

As a rare but potentially serious and idiosyncratic adverse drug reaction, DILI has been proven to be associated with genetic polymorphisms in genes involving in drug metabolism and transport, immune reaction and antioxidant response $\mathrm{e}^{12}$. However, the association between genetic variations of $A B C B 11$ and risk of DILI is still unclear with inconsistent results from previous studies. Lang et al. found that polymorphism in rs2287622 was associated with drug-induced cholestasis in Caucasian patients ${ }^{11}$ while Kagawa et al. reported no contribution of the same polymorphism in Japanese patients with drug-induced cholestasis ${ }^{13}$. Furthermore, the study of Ulzurrun et al. showed that the $\mathrm{C}$ allele in the $A B C B 11 \mathrm{rs} 2287622$ polymorphism was associated with increased risk of hepatocellular type DILI for drugs containing a carbocyclic system with aromatic rings ${ }^{10}$.

${ }^{1}$ Department of Epidemiology and Biostatistics, School of Public Health, Peking University Health Science Centre, Beijing, China. ${ }^{2}$ Department of Epidemiology, School of Public Health, Nanjing Medical University, Nanjing, China. ${ }^{3}$ Department of Clinical Epidemiology and Biostatistics, McMaster University, Hamilton, Canada. ${ }^{4}$ Clinical Research Division, Peking University Institute of Mental Health, and Key Laboratory for Mental Health, Ministry of Health, Beijing, China. ${ }^{5}$ Center for Tuberculosis Control and Prevention, Chinese Center for Disease Control and Prevention, Beijing, China. Correspondence and requests for materials should be addressed to S.Z. (email: siyan-zhan@bjmu. edu.cn) 


\begin{tabular}{|l|c|c|c|}
\hline Characteristic & $\begin{array}{c}\text { Patients with ATDILI } \\
(\mathbf{N}=89)\end{array}$ & $\begin{array}{c}\text { Patients without } \\
\text { ATDILI (N=356) }\end{array}$ & P value \\
\hline Sex (male/female) & $65 / 24$ & $260 / 96$ & \\
\hline Treatment history (primary/re-treatment) & $78 / 11$ & $312 / 44$ & \\
\hline Age $($ years) & $43.7 \pm 16.4(20.0-80.0)$ & $43.6 \pm 16.4(17.0-84.0)$ & $0.742^{\mathrm{c}}$ \\
\hline Weight $(\mathrm{kg})^{\mathrm{a}}$ & $53.2 \pm 8.0(38.0-80.0)$ & $53.5 \pm 7.4(31.0-84.0)$ & $0.748^{\mathrm{c}}$ \\
\hline BMI $\left(\mathrm{kg} / \mathrm{m}^{2}\right)^{\mathrm{a}}$ & $19.5 \pm 2.3(14.0-27.7)$ & $19.4 \pm 2.3(13.5-27.0)$ & $0.959^{\mathrm{c}}$ \\
\hline Baseline value & & & \\
\hline AST $(\mathrm{U} / \mathrm{L})^{\mathrm{b}}$ & $24.8(17.2-32.6)$ & $21.4(15.3-27.0)$ & $0.283^{\mathrm{d}}$ \\
\hline ALT $(\mathrm{U} / \mathrm{L})^{\mathrm{b}}$ & $16.9(10.8-26.3)$ & $16.0(10.4-22.0)$ & $0.087^{\mathrm{d}}$ \\
\hline TBIL $(\mu \mathrm{mol} / \mathrm{L})^{\mathrm{b}}$ & $9.5(7.5-13.7)$ & $9.7(7.4-12.5)$ & $1.000^{\mathrm{d}}$ \\
\hline${ }^{\mathrm{d} u r i n g} \operatorname{treatment}($ peak value $)$ & & & \\
\hline AST $(\mathrm{U} / \mathrm{L})^{\mathrm{b}}$ & $95.1(60.6-174.7)$ & $23.7(16.7-29.0)$ & $<0.001^{\mathrm{d}}$ \\
\hline ALT $(\mathrm{U} / \mathrm{L})^{\mathrm{b}}$ & $121.0(88.2-183.6)$ & $17.0(11.6-23.2)$ & $<0.001^{\mathrm{d}}$ \\
\hline TBIL $(\mu \mathrm{mol} / \mathrm{L})^{\mathrm{b}}$ & $14.3(11.4-18.0)$ & $9.7(6.8-13.7)$ & $<0.001^{\mathrm{d}}$ \\
\hline
\end{tabular}

Table 1. Characteristics of patients with and without ATDILI. Abbreviations: BMI, body mass index; ALT,

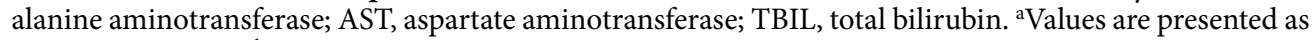

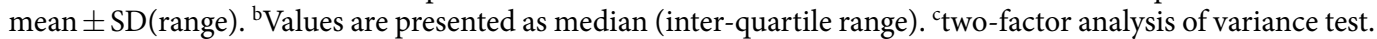
${ }^{\mathrm{d}}$ Median test.

\begin{tabular}{|l|c|c|c|c|c|}
\hline SNP & Chromosome position $^{\mathbf{a}}$ & Position & SNP name & MAF $^{\mathbf{b}}$ & HWE $_{\text {p-value }}$ \\
\hline rs496550 & $2: 168923202$ & $3^{\prime}$ UTR & $420 \mathrm{~A}>\mathrm{G}$ & 34.9 & 0.472 \\
\hline rs2287622 & $2: 168973818$ & Exon & $1331 \mathrm{~T}>\mathrm{C}$ & 32.1 & 0.415 \\
\hline rs3770592 & $2: 168981530$ & Intron & $\begin{array}{c}1084- \\
1551 \mathrm{~A}>\mathrm{T}\end{array}$ & 23.2 & 0.867 \\
\hline rs2287616 & $2: 168990902$ & Exon & $807 \mathrm{~T}>\mathrm{C}$ & 38.4 & 0.265 \\
\hline
\end{tabular}

Table 2. Information on four genotyped tag SNPs in ABCB11 gene. ${ }^{a}$ NCBI SNP database on GRCh37. p10 assembly. ${ }^{b} \mathrm{MAF}$ (Minor Alleles Frequency) for Han Chinese in Beijing in the HapMap database. ${ }^{\mathrm{c}}$ HardyWeinberg equilibrium (HWE) P-value in the control group.

As one of the most prevalent DILI, anti-tuberculosis drugs (ATDs) induced liver injury (ATDILI) is of importance due to relatively high incidence and large disease burden of tuberculosis (TB), which posed a considerable challenge to clinicians and researchers. The mechanism of ATDILI is poorly understood and whether genetic variants of $A B C B 11$ contribute to its susceptibility is seldom investigated. Vitro study has demonstrated that ATDs administration significantly down-regulated the expression of BSEP in liver ${ }^{14}$, therefore, it is reasonable to speculate that genetic variations in $A B C B 11$ may play a role in the development of ATDILI by altering the expression of BSEP and disturbing the transport of bile acids ${ }^{15}$. Therefore, the present study is aimed to explore the association between $A B C B 11$ polymorphisms and the risk of ATDILI in Chinese population.

\section{Results}

Baseline characteristics. 89 patients with ATDILI and 356 matched controls were included in the study. Their baseline characteristics were summarized in Table 1. No significant difference was observed between the two groups regarding demographic parameters, including age, weight or body mass index, and baseline values of liver biochemical parameters (all $P>0.05$ ). However, the peak AST, ALT, and TBIL levels were significantly higher in cases than those in controls during the treatment $(P<0.001)$.

Genotype analysis. The observed genotype frequencies for the selected SNPs in the controls were all in Hardy-Weinberg equilibrium ( $P>0.05$, respectively) (Table 2$)$. The genotype distributions of the four SNPs in $A B C B 11$ gene were presented in Table 3 . None of them was significantly associated with the developmentof ATDILI.

Subgroup analysis. According to diagnostic standard proposed in international consensus meeting, ATDILI could be categorized as cholestatic, mixed and hepatocellular liver injury based on the ratio of ALT and alkaline phosphatase (ALP). In this study, 28 cases with ALP data and were dichotomized into hepatocellular group or cholestatic/mixed group, with the rest of 61 cases classified as unclear type. The association between selected SNPs and the risk of ATDILI among different diagnoses of liver injury were shown in Table 4 . The frequency of rs2287616 in $A B C B 11$ gene were significantly different between cholestatic/mixed patients and matched controls under dominant model and addictive model $(\mathrm{OR}=3.84,95 \% \mathrm{CI}: 1.16-12.75, P=0.028$ and $\mathrm{OR}=2.51,95 \%$ CI:1.12-5.62, $P=0.025$, respectively), however the significance disappeared after Bonferroni correction.

Supplementary analyses. A comparison of ALT, AST and TBIL in TB patients with different genotypes of each SNP was summarized in Fig. 1. There were no statistically significant difference in the levels of ALT and AST 


\begin{tabular}{|c|c|c|c|c|c|c|c|}
\hline Genotype & Cases N(\%) & Controls N(\%) & OR $(95 \% \mathrm{CI})$ & P value & Model & OR $(95 \% \mathrm{CI})$ & Pvalue \\
\hline \multicolumn{8}{|l|}{ rs 496550} \\
\hline GG & $38(43.7)$ & $150(43.4)$ & 1 (reference) & & Dom & $0.95(0.60-1.53)$ & 0.844 \\
\hline AG & $38(43.7)$ & 151(43.6) & $0.99(0.60-1.64)$ & 0.965 & $\operatorname{Rec}$ & $0.85(0.42-1.76)$ & 0.668 \\
\hline $\mathrm{AA}$ & $11(12.6)$ & $45(13.0)$ & $0.85(0.40-1.81)$ & 0.673 & Add & $0.94(0.67-1.32)$ & 0.727 \\
\hline \multicolumn{8}{|l|}{ rs 2287622} \\
\hline $\mathrm{CC}$ & $44(50.0)$ & $185(52.3)$ & 1 (reference) & & Dom & $1.06(0.67-1.68)$ & 0.810 \\
\hline $\mathrm{CT}$ & $37(42.0)$ & $146(41.2)$ & $1.02(0.63-1.65)$ & 0.925 & $\operatorname{Rec}$ & $1.31(0.53-3.21)$ & 0.557 \\
\hline TT & $7(8.0)$ & $23(6.5)$ & $1.32(0.53-3.33)$ & 0.553 & Add & $1.09(0.75-1.58)$ & 0.666 \\
\hline \multicolumn{8}{|l|}{ rs3770592 } \\
\hline $\mathrm{AA}$ & $54(60.7)$ & $217(61.3)$ & 1 (reference) & & Dom & $1.02(0.63-1.64)$ & 0.949 \\
\hline AT & $32(36.0)$ & $121(34.2)$ & $1.04(0.64-1.71)$ & 0.862 & $\operatorname{Rec}$ & $0.78(0.23-2.69)$ & 0.692 \\
\hline TT & $3(3.3)$ & $16(4.5)$ & $0.79(0.23-2.77)$ & 0.714 & Add & $0.98(0.66-1.47)$ & 0.931 \\
\hline \multicolumn{8}{|l|}{ rs2287616 } \\
\hline TT & $37(43.0)$ & $178(50.3)$ & 1 (reference) & & Dom & $1.31(0.81-2.11)$ & 0.266 \\
\hline $\mathrm{CT}$ & $38(44.2)$ & $152(42.9)$ & $1.18(0.71-1.97)$ & 0.532 & $\operatorname{Rec}$ & $1.88(0.86-4.08)$ & 0.112 \\
\hline CC & $11(12.8)$ & $24(6.8)$ & $2.01(0.90-4.49)$ & 0.090 & Add & $1.33(0.93-1.90)$ & 0.123 \\
\hline
\end{tabular}

Table 3. $A B C B 11$ polymorphisms in patients with and without ATDILI. Abbreviations: Dom, dominant model; Rec, recessive model; Add, additive model.

\begin{tabular}{|c|c|c|c|c|c|c|c|}
\hline \multirow[b]{2}{*}{ SNP } & \multirow[b]{2}{*}{ Model } & \multicolumn{2}{|c|}{ Hepatocellular $(\mathrm{N}=11)$} & \multicolumn{2}{|c|}{ Cholestatic/Mixed(N=17) } & \multicolumn{2}{|c|}{ Unclear $(\mathrm{N}=61)$} \\
\hline & & OR(95\%CI) & P value & OR(95\%CI) & P value & OR(95\%CI) & P value \\
\hline \multirow{3}{*}{ rs 496550} & Dom & $0.53(0.14-1.98)$ & 0.345 & $1.53(0.49-4.74)$ & 0.461 & $0.93(0.53-1.66)$ & 0.814 \\
\hline & $\operatorname{Rec}$ & $0.66(0.06-7.11)$ & 0.731 & $3.08(0.57-16.73)$ & 0.193 & $0.67(0.28-1.61)$ & 0.368 \\
\hline & Add & $0.64(0.23-1.79)$ & 0.394 & $1.61(0.70-3.70)$ & 0.263 & $0.88(0.58-1.33)$ & 0.538 \\
\hline \multirow{3}{*}{ rs 2287622} & Dom & $2.12(0.57-7.87)$ & 0.262 & $0.64(0.23-1.80)$ & 0.402 & $1.08(0.61-1.91)$ & 0.803 \\
\hline & $\operatorname{Rec}$ & $3.56(0.20-64.34)$ & 0.391 & $0.48(0.05-4.23)$ & 0.506 & $1.59(0.53-4.77)$ & 0.411 \\
\hline & Add & $2.14(0.67-6.83)$ & 0.199 & $0.68(0.30-1.55)$ & 0.357 & $1.14(0.71-1.82)$ & 0.594 \\
\hline \multirow{3}{*}{ rs3770592 } & Dom & $1.02(0.25-4.16)$ & 0.975 & $0.94(0.31-2.89)$ & 0.911 & $1.06(0.60-1.88)$ & 0.848 \\
\hline & $\operatorname{Rec}$ & NA & NA & NA & NA & NA & NA \\
\hline & Add & $0.85(0.24-3.07)$ & 0.807 & $0.83(0.31-2.20)$ & 0.702 & $1.08(0.66-1.75)$ & 0.769 \\
\hline \multirow{3}{*}{ rs2287616 } & Dom & $0.35(0.07-1.67)$ & 0.186 & $3.84(1.16-12.75)$ & 0.028 & $1.17(0.66-2.07)$ & 0.590 \\
\hline & $\operatorname{Rec}$ & NA & NA & $3.27(0.68-15.73)$ & 0.140 & $1.85(0.71-4.79)$ & 0.206 \\
\hline & Add & $0.36(0.08-1.62)$ & 0.181 & $2.51(1.12-5.62)$ & 0.025 & $1.24(0.80-1.94)$ & 0.335 \\
\hline
\end{tabular}

Table 4. $A B C B 11$ polymorphisms in patients with and without ATDILI among different diagnoses of liver injury. Abbreviations: Dom, dominant model; Rec, recessive model; Add, additive model; NA, not applicable.

between the various genotypes of selected SNPs. However, in rs496550 and rs2287616 SNPs, significant associations were observed in TBIL values among patients with different genotypes $(P=0.015$ and 0.017 , respectively). Of note, patients carrying a TT genotype in rs 496550 or a CC genotype in rs 2287616 had a higher value of TBIL compared with other genotypes under the treatment of ATDs.

When analyzing genotype distribution of the selected SNPs polymorphism in patients classified by adverse reactions symptoms, we found that rs2287616 CC genotype distributed differently in gastrointestinal disorders ( $31.6 \%$ cases vs $5.6 \%$ controls), arthralgia ( $40.0 \%$ cases vs $8.9 \%$ controls) and pruritus $(31.6 \%$ cases vs $5.6 \%$ controls) (Table 5). The genotype distribution of rs496550, rs2287622 and rs3770592 based on different symptoms were presented in supplementary table S1, S2 and S3, none of the genotypes showed significant difference except for rs496550 in nausea/vomiting $\left(\chi^{2}=10.056, P=0.007\right)$.

\section{Discussion}

In this study, we investigated the association between polymorphisms of $A B C B 11$ and the development of ATDILI among Chinese population. The results shows that though there was no significant differences in genotype frequencies between patients with and without ATDILI in general, the polymorphisms of rs2287616 in $A B C B 11$ gene were significantly associated with cholestatic/mixed liver injury in the subgroup analysis. Besides, patients carrying the CC genotype of rs2287616 had a higher value of TBIL compared with CT/TT genotype. In addition, significantly increased frequencies of rs2287616 CC genotype were noticed in some adverse effects symptoms including gastrointestinal disorders, arthralgia and pruritus. These findings suggested that the 


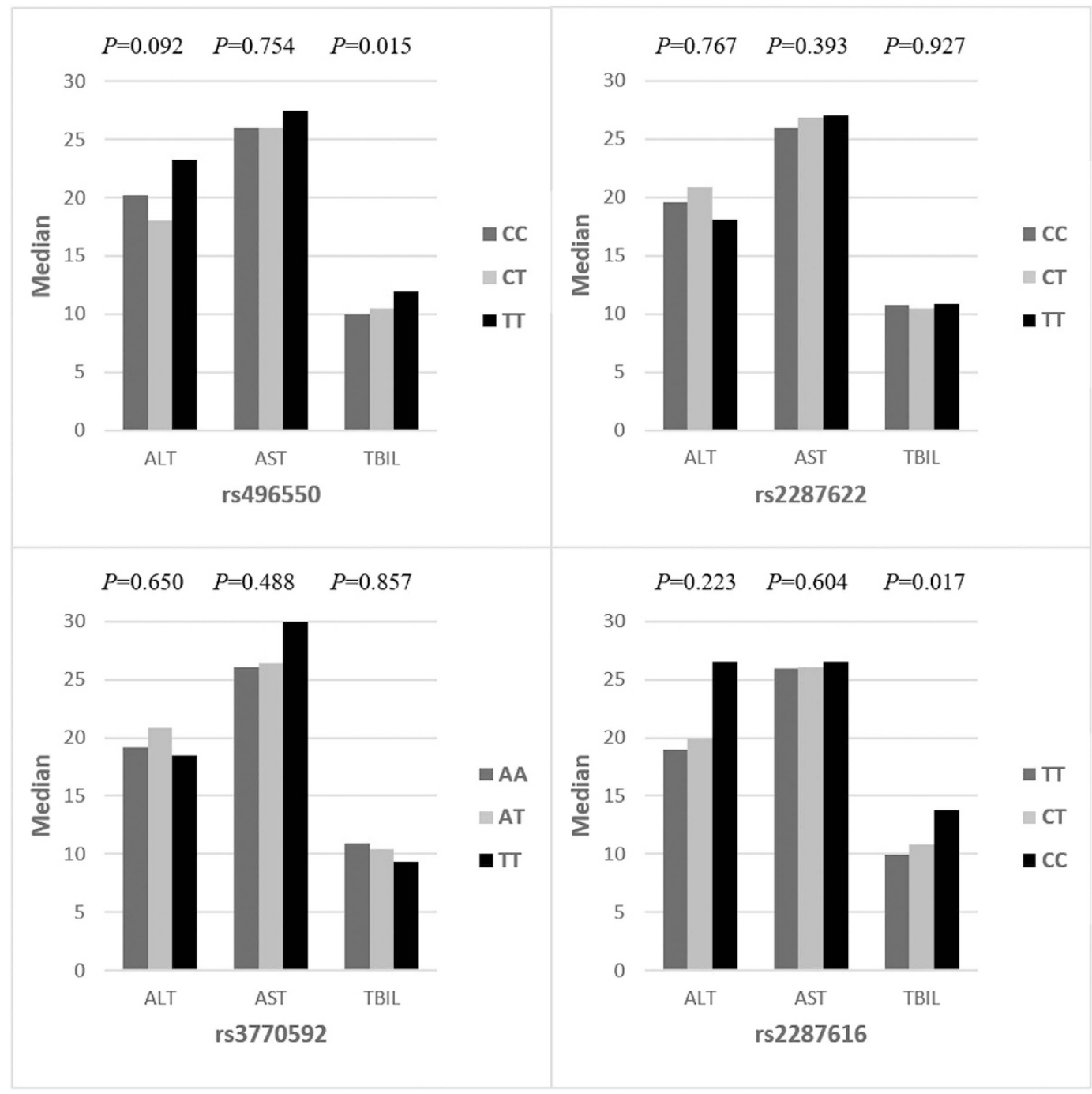

Figure 1. Median values of ALT, AST and TB in different genotype groups of $A B C B 11$ gene. The values are shown as U/L for ALT and AST and $\mu \mathrm{mol} / \mathrm{L}$ for TBIL. Abbreviations: ALT, alanine aminotransferase; AST, aspartate aminotransferase; TBIL, total bilirubin.

\begin{tabular}{|c|c|c|c|c|c|c|}
\hline \multirow{2}{*}{\multicolumn{2}{|c|}{ Symptoms }} & \multicolumn{3}{|c|}{ Genotype distribution } & \multirow{3}{*}{$\frac{\chi^{2}}{1.093}$} & \multirow{3}{*}{$\frac{\text { P value }}{0.579}$} \\
\hline & & \multirow{2}{*}{\begin{tabular}{|c|} 
TT N(\%) \\
$2(28.6)$
\end{tabular}} & \multirow{2}{*}{\begin{tabular}{|c|} 
CT N(\%) \\
$4(57.1)$
\end{tabular}} & \multirow{2}{*}{$\begin{array}{c}\text { CC N(\%) } \\
1(14.3)\end{array}$} & & \\
\hline Fever & Yes & & & & & \\
\hline rever & No & $23(47.9)$ & $18(37.5)$ & $7(14.6)$ & & \\
\hline \multirow{2}{*}{ Dizziness/headache } & Yes & $9(37.5)$ & $11(45.8)$ & $4(16.7)$ & 1.087 & 0.581 \\
\hline & No & $16(51.6)$ & $11(35.5)$ & $4(12.9)$ & & \\
\hline \multirow{2}{*}{ Gastrointestinal disorders ${ }^{\mathrm{a}}$} & Yes & $5(26.3)$ & $8(42.1)$ & $6(31.6)$ & 8.162 & 0.017 \\
\hline & No & $20(55.6)$ & $14(38.9)$ & $2(5.6)$ & & \\
\hline \multirow{2}{*}{ Nausea/ vomiting } & Yes & $15(38.5)$ & $19(48.7)$ & $5(12.8)$ & 4.264 & 0.119 \\
\hline & No & $10(62.5)$ & $3(18.8)$ & $3(18.8)$ & & \\
\hline \multirow{2}{*}{ Fatigue/lethargy/insomnia } & Yes & $6(30.0)$ & $9(45.0)$ & $5(25.0)$ & 4.209 & 0.122 \\
\hline & No & $19(54.3)$ & $13(37.1)$ & $3(8.6)$ & & \\
\hline \multirow{2}{*}{ Arthralgia } & Yes & $1(10.0)$ & $5(50.0)$ & $4(40.0)$ & 9.130 & 0.010 \\
\hline & No & $24(53.3)$ & $17(37.8)$ & $4(8.9)$ & & \\
\hline \multirow{2}{*}{ Pruritus } & Yes & $6(31.6)$ & $7(36.8)$ & $6(31.6)$ & 7.092 & 0.029 \\
\hline & No & $19(52.8)$ & $15(41.7)$ & $2(5.6)$ & & \\
\hline
\end{tabular}

Table 5. Distribution of $A B C B 11$ rs2287616 genotype in ATDILI patients with various symptoms. ${ }^{\text {anclude }}$ diarrhea, abdominal pain and abdominal distension period. 
polymorphisms of $A B C B 11$ gene might be associated with ATDs-induced cholestatic liver injury in Chinese TB patients.

BSEP is responsible for the bile salt-dependent bile flow and is regarded as an important gene in many liver diseases including DILI ${ }^{2}$. Previous studies have identified a common BSEP polymorphism V44A (1331T $>$ C, rs2287622) as genetic risk factor for DILI in Caucasian ${ }^{10,11}$ In the present study, we tested the same SNP in Chinese patients, however, no significant association was found between rs2287622 polymorphism and the development of DILI, which was similar to the study conducted in Japan ${ }^{13}$. Since genetic associations were ethnicity-specific and drug-specific, the association of $A B C B 11$ rs2287622 genetic variations and DILI should be investigated in other populations and other drugs.

Despite of rs2287622, a new SNP, rs2287616 was found to be associated with cholestatic/mixed liver injury in this study. Limited by the sample size of cholestatic pattern of ATDILI, supplementary analyses were taken to provide indirect evidence to the association. The signs and symptoms of cholestatic liver disease may include hyperbilirubinemia, gastrointestinal disorders, fatigue, pruritus, arthralgia, and so on. Therefore, we compare the value of liver function tests and the symptoms of common adverse drug effects in ATDILI patients with different genotypes of $A B C B 11$ rs2287616. The results showed that polymorphisms in rs2287616 had a significant association with increased TBIL, gastrointestinal disorders, arthralgia and itching/rash, indicating the role of genetic variations of $A B C B 11$ in the development of ATDs-induced cholestatic liver injury. Besides, supplementary analyses revealed the association between rs496550 and an evaluated TBIL as well as the symptom of nausea/ vomiting, which pointed the importance of $A B C B 11$ polymorphisms in ATDs-induced adverse reaction.

The $A B C B 11$ gene encoding human BSEP consists of 28 exons (including non-coding exon 1) and spans approximately $108 \mathrm{~kb}$ on the long arm of chromosome $2(2 \mathrm{q} 24-31)^{16} \mathrm{Rs} 2287616$ is a synonymous mutation on exon 9 and rs496550 is located on 3'URT. Though the function and mechanism of these two SNPs is poorly understood, as they are both tag SNPs that represent a group of SNPs with high linkage disequilibrium in regions of $A B C B 11$ gene, it indicates a possible role of genetic variations within the blocks of $A B C B 11$ in ATDs-induced cholestatic liver injury. Moreover, vitro study has proven that INH/RMP administration significantly down-regulated the expression of BSEP in liver of mice ${ }^{14}$. Therefore, the genetic susceptibility to ATDILI may be explained by the decreased expression of BSEP in the presence of polymorphisms in $A B C B 11^{17}$, leading to increased bile salt concentrations in hepatocytes, which contributes to the development of liver injury.

The emerging role of hepatic transporters in regulating the movement of endogenous and exogenous chemicals (e.g., bile acids and drugs) across cellular and tissue membranes is critical in determining the pathophysiology of liver disease ${ }^{18}$. In this study, we focus on the gene that regulates the transport of bile acids. The different findings in subgroup analysis pinpointed the significant contribution of BSEP to the complexity of ATDILI. The mechanism of ATDILI varies among different patterns of liver injury, which consequently changes the genetic susceptibility to the disease. Future investigations should pay attention to the specific mechanism of each pattern and evaluate the genetic association respectively.

The major strength of the present study is that it was a case-control study nested in the ACADS cohort, which meant this study was less likely to be subjected to recall bias and other possible biases introduced by self-selection of patients. Secondly, each case was verified by experts strictly to minimize the diagnosis misclassification. In addition, we performed a 1:4 matching to control effects of some potential confounders as well as to increase the statistical power.

However, the results of this study are limited by the small sample size in cholestatic/mixed patients. Because the information of ALP was not included for all patients, only a few cases could be identified as hepatocellular, cholestatic or mixed. As a result, we have to combine cholestatic group and mixed group together in the subgroup analysis, and take supplementary analyses to further explain the results. Besides, the ATDs were used as a combination, thus it is difficult to identify the association of each drug and its adverse reaction.

In conclusion, we found that genetic polymorphism of $A B C B 11$ might contribute to ATDs-induced cholestatic liver injury in Chinese population. Studies in larger, varied populations are required to confirm these findings.

\section{Methods}

Study population. Anti-TB treatment patients were recruited from ADACS cohort ${ }^{19}$, which is a prospective longitudinal study of ATD induced adverse reactions based on community population. From October 2007 to June 2008, a total of 4488 newly diagnosed TB patients were recruited from four provinces in China. All patients were treated with a standard chemotherapy regimen with a combination of ATDs including isoniazid (INH), rifampicin (RMP), pyrazinamide (PZA), ethambutol (EMB) and/or streptomycin (SM) for six to nine months ${ }^{20}$. Liver function tests were performed within 2 months of the initiation of treatment in all patients or whenever the patients exhibited symptoms of suspected hepatitis (e.g. anorexia, nausea, vomiting, malaise, teacoloured urine). A total of 4304 patients finished the follow-up. This study was approved by the Ethics Committee of Center for Tuberculosis Control and Prevention of China and Health Science Center of Peking University. Written informed consent was obtained from every participant or surrogate before enrolment. The present study was conducted in accordance with the Declaration of Helsinki Principles.

ATDILI was defined as: (i) an increase in alanine aminotransferase (ALT) levels greater than two-times of the upper limit of normal (ULN) with/without a combined increase in aspartate aminotransferase (AST) and total bilirubin (TBIL) levels provided one of them was greater than two-times of ULN during the treatment ${ }^{21}$; (ii) causality assessment result was certain, probable or possible based on the WHO Uppsala Monitoring Center system ${ }^{22}$. All suspected hepatitis patients were reviewed by experts from Chinese State Food and Drug Administration. Patients with any of the following were excluded from the present study: (i) abnormal serum ALT, AST or TBIL levels before anti-TB treatment; (ii) carriers of the hepatitis B or C virus; (iii) alcoholic liver disease or habitual alcohol drinking; (iv) the concomitant use of hepatotoxic drugs; and (v) a history of chronic liver disease or systemic diseases that may cause liver dysfunction. Among the remained patients, those fulfilled 
the criteria of ATDILI were assigned into the case group. For each ATDILI case, four controls were randomly selected and matched with age (within 5 years old), sex, treatment history, disease severity, drug dosage and place of sample collection.

Selection of SNPs. A tag-SNP approach was used to select the SNPs. All eligible SNPs in the gene region including 2-kb upstream were obtained from the Chinese Han population data available on the International HapMap website (http://hapmap.ncbi.nlm.nih.gov, HapMap Genome Browser release \#24). The tag SNPs were selected using Haploview 4.2 software based on the following criteria: i) minor allele frequency more than 0.10 ; ii) $\mathrm{r}^{2}$ of pairwise linkage disequilibrium more than 0.80. As a result, four SNPs (rs496550, rs2287622, rs3770592 and rs2287616) were chosen for genotyping (Table 2).

Laboratory procedures. Blood sample was collected from each patient during laboratory examination after their recruitment. Then DNA was extracted and genotyped by TaqMan allelic discrimination technology on the ABI 7900 Real-Time PCR System (Applied Biosystems, Foster City, CA) ${ }^{23}$. The primers and probes for each SNP were designed by Nanjing Steed BioTechnologies Co., Ltd. Genotyping was performed by blinding the case or control status, and with a positive control of a DNA sample with a known heterozygous genotype in each test. More than $10 \%$ of the samples were repeated using the same assay and the results were $100 \%$ concordant. The overall call rate of genotyping was more than $98 \%$.

Statistical analysis. Continuous variables were described as mean \pm standard deviation (SD) or median (IQR, inter-quartile range) and differences between groups were analyzed by two-factor analysis of variance test or non-parametric test. Categorized variables were described as percentage and analyzed using the $\chi^{2}$ test. Hardy-Weinberg equilibrium was tested by a goodness-of-fit $\chi^{2}$ test. Genotype frequencies were compared in the ATDILI patient and control groups by multivariate conditional logistic regression analysis, with weight and usage of liver-protective drug as covariates. Kruskal-Waillis test was used to compare the distribution of mean AST, ALT and TBIL values in the different genotype patterns. The associations between selected SNPs and the risk of ATDILI were estimated by odds ratios (OR) with $95 \%$ confidence intervals (CIs). Three different analysis models, dominant (Dom), recessive (Rec) and addictive (Add), were used to compare genotype frequencies. A two-tailed P-value less than 0.05 was considered to be statistically significant. Considering the potential false positive rate incurred by multiple comparisons of SNPs, we applied the Bonferroni correction method to adjust the P value. The statistical analyses were performed with SPSS for Windows (version 19.0, IBM Inc.).

\section{References}

1. Kubitz, R., Droge, C., Kluge, S., Stindt, J. \& Haussinger, D. Genetic variations of bile salt transporters. Drug Discov Today Technol 12, e55-67 (2014).

2. Kubitz, R., Droge, C., Stindt, J., Weissenberger, K. \& Haussinger, D. The bile salt export pump (BSEP) in health and disease. Clin Res Hepatol Gastroenterol 36, 536-53 (2012).

3. Keitel, V. et al. Expression and localization of hepatobiliary transport proteins in progressive familial intrahepatic cholestasis. Hepatology 41, 1160-72 (2005).

4. Strautnieks, S.S. et al. A gene encoding a liver-specific ABC transporter is mutated in progressive familial intrahepatic cholestasis. Nat Genet 20, 233-8 (1998).

5. Jansen, P.L. et al. Hepatocanalicular bile salt export pump deficiency in patients with progressive familial intrahepatic cholestasis. Gastroenterology 117, 1370-9 (1999).

6. van Mil, S.W. et al. Benign recurrent intrahepatic cholestasis type 2 is caused by mutations in ABCB11. Gastroenterology 127, 379-84 (2004).

7. Kubitz, R., Keitel, V., Scheuring, S., Kohrer, K. \& Haussinger, D. Benign recurrent intrahepatic cholestasis associated with mutations of the bile salt export pump. J Clin Gastroenterol 40, 171-5 (2006).

8. Dixon, P.H. et al. Contribution of variant alleles of ABCB11 to susceptibility to intrahepatic cholestasis of pregnancy. Gut 58, 537-44 (2009).

9. Meier, Y. et al. Increased susceptibility for intrahepatic cholestasis of pregnancy and contraceptive-induced cholestasis in carriers of the $1331 \mathrm{~T}>\mathrm{C}$ polymorphism in the bile salt export pump. World J Gastroenterol 14, 38-45 (2008).

10. Ulzurrun, E. et al. Role of chemical structures and the $1331 \mathrm{~T}>\mathrm{C}$ bile salt export pump polymorphism in idiosyncratic drug-induced liver injury. Liver Int 33, 1378-85 (2013).

11. Lang, C. et al. Mutations and polymorphisms in the bile salt export pump and the multidrug resistance protein 3 associated with drug-induced liver injury. Pharmacogenet Genomics 17, 47-60 (2007).

12. Daly, A.K. Drug-induced liver injury: past, present and future. Pharmacogenomics 11, 607-11 (2010).

13. Kagawa, T. et al. No contribution of the ABCB11 p.444A polymorphism in Japanese patients with drug-induced cholestasis. Drug Metab Dispos 43, 691-7 (2015).

14. Guo, Y.X. et al. The inhibition of hepatic bile acids transporters Ntcp and Bsep is involved in the pathogenesis of isoniazid/ rifampicin-induced hepatotoxicity. Toxicol Mech Methods 25, 382-7 (2015).

15. Ho, R.H. et al. Polymorphic variants in the human bile salt export pump (BSEP; ABCB11): functional characterization and interindividual variability. Pharmacogenet Genomics 20, 45-57 (2010).

16. Kim, S.R. et al. Genetic variations of the ABC transporter gene ABCB11 encoding the human bile salt export pump (BSEP) in a Japanese population. Drug Metab Pharmacokinet 24, 277-81 (2009).

17. Garzel, B. et al. The role of bile salt export pump gene repression in drug-induced cholestatic liver toxicity. Drug Metab Dispos 42, 318-22 (2014).

18. Corsini, A. \& Bortolini, M. Drug-induced liver injury: the role of drug metabolism and transport. J Clin Pharmacol 53, 463-74 (2013).

19. Xia, Y.Y. et al. Design of the anti-tuberculosis drugs induced adverse reactions in China National Tuberculosis Prevention and Control Scheme Study (ADACS). BMC Public Health 10, 267 (2010).

20. An expanded DOTS framework for effective tuberculosis control. Int J Tuberc Lung Dis 6, 378-88 (2002).

21. Benichou, C. Criteria of drug-induced liver disorders. Report of an international consensus meeting. J Hepatol 11, 272-6 (1990).

22. World Health Organization. The use of the WHO-UMC system for standardized case causality assessment. Uppsala: The Uppsala Monitoring Centre. (2005).

23. Teuber, M., Wenz, M.H., Schreiber, S. \& Franke, A. GMFilter and SXTestPlate: software tools for improving the SNPlex genotyping system. BMC Bioinformatics 10, 81 (2009). 


\section{Acknowledgements}

This study was funded by the National Natural Science Foundation of China (81072387), Beijing Natural Science Foundation (7111006). The authors thank all cooperating organizations for their support.

\section{Author Contributions}

Study design (S.Z. and D.C.), data collection and cleaning (Y.X., Z.Y. and S.W.), gene detection (R.C., J.W. and S.T.), data analysis (Y.Z., X.L. and S.T.), manuscript writing (R.C. and J.W.)

\section{Additional Information}

Supplementary information accompanies this paper at http://www.nature.com/srep

Competing financial interests: The authors declare no competing financial interests.

How to cite this article: Chen, R. et al. Role of polymorphic bile salt export pump (BSEP, ABCB11) transporters in anti-tuberculosis drug-induced liver injury in a Chinese cohort. Sci. Rep. 6, 27750; doi: 10.1038/srep27750 (2016).

(c) (i) This work is licensed under a Creative Commons Attribution 4.0 International License. The images or other third party material in this article are included in the article's Creative Commons license, unless indicated otherwise in the credit line; if the material is not included under the Creative Commons license, users will need to obtain permission from the license holder to reproduce the material. To view a copy of this license, visit http://creativecommons.org/licenses/by/4.0/ 